\title{
Web application for an occupancy plan in a passenger railway station
}

\author{
M. Bažant \& J. Žampach \\ Faculty of Electrical Engineering and Informatics, \\ University of Pardubice, Czech Republic
}

\begin{abstract}
This paper deals with the problem of track occupation diagram management for passenger railway stations. It summarizes the current techniques of diagrams creation, its usefulness for railway dispatchers and other managers. The practical aim of this work is to design and implement a web application that would partially allow to automate the process of diagram creation and to provide an interactive tool for operational traffic control with an option to make dynamic changes.
\end{abstract}

Keywords: track occupation diagram, railway transport, web application.

\section{Introduction}

The main aim of this paper is to present an overview for the problem of creation and use of a track occupation diagram for larger passenger railway stations and describe the proposed software tool that would allow automate the process of diagram creation and provide an interactive tool for train dispatchers.

A track occupation diagram is used as a tool for providing valuable information for dispatchers that are responsible for controlling railway traffic at the level of railway station. It visually completes primary sources of information. Importance of track occupation diagram is in the:

- preparation of train traffic diagram (TTD) - an overview of whether the planned number of trains in a given time period is bearable.

- $\quad$ support for determining running tracks for all trains in the TTD.

- solution of exceptional situations mainly consisting of train delays (e.g. when a train is running late and currently there is waiting another train on the same track). 
Another benefit is in particular in the case of solving nonstandard situations such as a track exclusion when all trains from incriminated track must be moved to another track. Track occupation diagram can also help to determine alternative track within a railway station.

Data for the diagram are obtained from the current timetable. Then the data are transformed to a specific graphic form that clearly concentrates the data related to the railway station. Data files needed to create a diagram mainly consist of a list of running tracks and a list of trains that are in timetable for the railway station during one day. Important attributes are train type, train number, planned station track and information about the time of arrival and departure for that particular train in the station. From this data set graphical structure of diagram is designed, whereas horizontally is displayed list of tracks in time range from 0.00 to 24.00. Trains on these tracks (axis) are displayed at their sojourn times whereas there is additional information such as time of arrival, time of departure, directions of train arrival and departure and a calendar when a train is scheduled.

Graphical design of the diagram is specific and corresponds to the established conventions of a given railway station. It may also be one of the reasons why there is currently no software tool to automate difficult manual assembly. In the early years diagrams were prepared handmade using colour ink. These days there are vector graphic tools that allow modifications to already made diagrams and that allow better handling with finished diagrams (whether it is a vector document or large format printing). The whole diagram assembly is unfortunately still manual work and often it does not differ from the way diagrams were prepared using pen and ink.

This paper presents a prototype of web application for the preparation and management of track occupation diagram. Application architecture is client-server, the client part of the application runs in a web browser whereas eliminating the need to install and update special software on all considered computers. Another advantage of this solution is the immediate availability of the current traffic situation for all dispatchers. This application provides a tool for interactive and agile assessment of actual situation.

\section{Track occupation diagram and its usage}

Track occupation diagram is a part of the Technological tools for train traffic diagram (TTD) managed by the regulation [1] and is developed for railway stations that are on the list which is published by RCP (regional traffic centre). It uses graphical form where there are all tracks occupied by regular trains and there is also information about duration of planned occupation time and also directions of trains (from where the train is coming and to which direction it is leaving the station). Diagram is approved by station master and then it is released as an official support for station dispatchers [1]. 


\subsection{Tool for dispatchers in passenger railway stations}

Track occupation diagram has a role as a supporting tool in both standard and exceptional situations during controlling traffic in a railway station. Unfortunately, in the current concept, diagram is a static tool that does not change in cases when some exceptional situations happen (diagram is printed and therefore it cannot be easily modified).

Construction of the diagram has benefits only for larger passenger railway station with heavy traffic because in smaller railway stations it is enough to have experienced dispatchers even during exceptional situations.

\subsection{Current state of creation of track occupation diagram}

It was already mentioned that there are currently no special software tools to help to create such diagrams and diagrams are not assembled centrally. Every bigger passenger railway station has its own conventions of designing their own diagram. This is due to the fact that there is no specialized software tool and also to the absence of internal regulation that would somehow regulate the problem of diagram creation. Data for diagrams are based on the current train traffic diagram and can be acquired from internal information systems (e.g. IS Kango that is maintained by SZDC - Czech railway infrastructure manager).

\subsection{Graphical layout of track occupation diagram}

Graphical layout corresponds to specific rules and conventions and is not regulated in [2]. Therefore the graphic layout of track occupation diagrams is slightly different for every single railway station and even captures different level of details.

Commonly the diagram is made up for generic day. This means that it includes all trains no matter of whether they operate every day or only on selected days within a week or a year. For this reason the result diagram might be confusing in busy times and therefore there are introduced additional graphical rules, e.g. it might be used different colour code for trains running less than three days a week etc.

The diagram consists of a list of tracks on y-axis and time from 0.00 am to $12.00 \mathrm{pm}$ on $\mathrm{x}$-axis (start point and end point of $\mathrm{x}$-axis merges). So there is a kind of network where combination of running track and time creates a clear position of train in the diagram. The train is shown in timeline (by rail) with line segment whereas length determines sojourn time at railway station. If a train only passes through the station there is only shown line segment crossing the running track (axis). If the train ends its journey in a station (or begins) the train is represented with an arrow ending (or beginning) on the running track. Specific "shape" of a train in the diagram is shown in the Figure 1. 


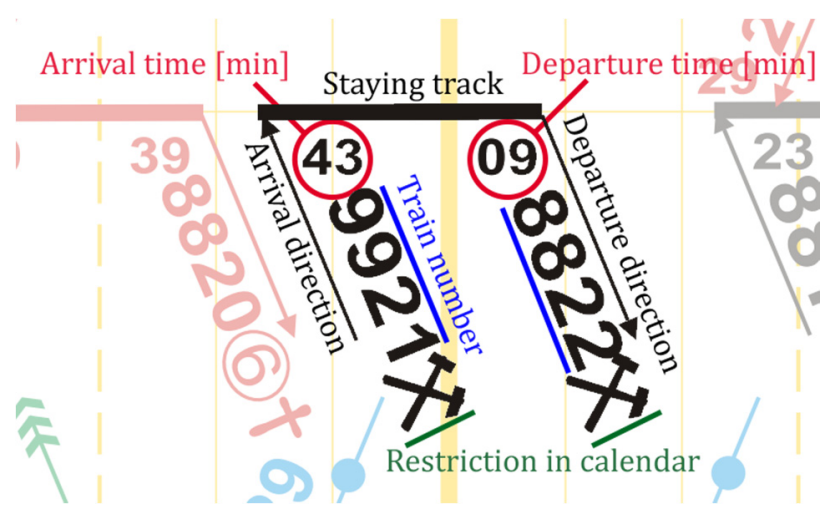

Figure 1: Example of a train in the track occupation diagram. Source: [3].

\subsection{Differences in track occupation diagrams in different railway stations}

From the analysis introduced in [2] it is obvious that conventions of creating diagram in bigger railway stations are very diverse (method of creating diagrams, method of drawing the symbols and shapes, etc.). E.g. for railway stations Prague main station and Pardubice main station there is used vector software Corel Draw $^{\text {TM }}$ whereas the railway station in Pilsen uses commonly available Microsoft Excel.

\section{Current state of track occupation diagram}

In the previous part of this paper there are introduced basic concepts of currently used track occupation diagrams. In this section there is presented detailed information of the currently used approaches and there will also be introduced the concept of the newly created solution.

\subsection{Advantages and disadvantages of current state}

The current situation of the development and use of diagram has been known for many years. Despite the fact that the creation of new diagram is manual and time-consuming work, this process is essential. The advantage of the current solution is a chance to include specific graphic elements for a railway station since these are not regulated by [1]. So the diagram is fully adapted and made-tomeasure for given railway station. The disadvantage of the current solution is complexity and slowness of the process of diagram creation when it is necessary to place all the elements to the diagram manually (whether in a graphic or spreadsheet software editor). There is also a risk of human factor which comprises in making errors. Also due to this risk, the plan is checked by station master of the railway station. After the revision the diagram is published as an official service tool for the needs of interested persons.

From the above text follows that it is not trivial to develop a comprehensive tool for the creation and management of the diagram which would satisfy current 
needs of the traffic dispatchers. But it is still possible to develop a compromise between clarity and usability. The software application has natural advantage of its functionality and the possibility of dynamic changes (in contrast to static diagrams). Any modifications made to the diagram are immediately accessible for all relevant employees.

\subsection{Currently used tools that are suitable for track occupation diagrams creation}

Currently there is no specialized software tool which is suitable for the diagram creation and management. Of course there are available some common applications that are not primarily intended for similar purposes but they could be used anyway such as Microsoft Excel or Corel Draw ${ }^{\mathrm{TM}}$.

There was already implemented one similar application as the thesis in the past [2]. The following section will briefly introduce it.

Application PLOK [1] is primarily used as a tool in the difficult process of track occupation diagram creation. It is a desktop application and its main goal is to provide a software platform for diagram designer that allows him to create advanced frame of diagram and set many parameters that are specific for the intended railway station before exporting or printing. The main features are e.g. choice of various colours, shapes of arrows, shapes of labels, etc. It is also possible to edit the constructed diagram (placing trains, changing train's numbers, etc.) and prepare it for export or printing. Application PLOK is based on the same source data as an application that is presented in this paper.

\subsection{Motivation and a proposal to solve the problem}

Application that is described in this paper does not have an ambition to create a diagram that is ready for printing and then used as a static tool. Ambition of this application is to create software tool that provides dynamic diagram that allows interactive changes to the diagram and that allows dispatchers to better utilize their potential (in comparison with the static concept of the diagram).

Presented application is trying to solve mentioned disadvantages of the static diagram. The application is designed to go beyond a static view of the diagram and develop a tool for traffic dispatchers with the ability to change diagram in various situations (track exclusion, train delay, etc.).

The concept of the application is as follows. The aim is to design an application that would facilitate assembly of the diagram based on source data and created diagram will be maintained and managed using this application. It allows dispatchers (depending on various user permissions) to use this application to dynamically modify the plan entering train delays, changing sojourn times for trains, changing default running tracks for trains and other minor parameters. Important changes can be done using interface in the diagram layout as well as in the list of all trains. The application offers interactive support for decision making for standard and also some exceptional situations. The application doesn't want to replace existing developed tool [1] because it would be very difficult to cover all established conventions. 


\section{Application development}

There are introduced main stages of the application development as well as application architecture and used technologies.

\subsection{Requirements}

Application design follows list of requirements. Functional requirements for interactive module of the diagram are mainly the ability to view the current state of the diagram, possibility to enter delays quickly, change sojourn time of a train in the diagram, alert for conflicts in the diagram after user action, the ability to filter the diagram by date (for clarity) and comfortable access to detailed information about a train placing cursor above a train in the diagram. There is also a requirement to distinguish between local changes (changes apply only for the logged in user) and global scope (changes take effect for all users). All changes are stored and it is possible to cancel them when it is needed. In the table view of trains there are requirements for an option to edit all train attributes, adding new trains, adding train exclusions, entering tracks exclusion and also to import new data from CSV file.

The main non-functional requirements include requirements for performance, reliability, scalability, security and usability of the system: client-server architecture on Java EE technology (client will use a web browser). Main part of the diagram will be constructed using vector graphics. System allows to work with user roles in three levels. Time accuracy is $0.5 \mathrm{~min}$.

\subsection{Architecture of the solution}

In practice there are more copies of track occupation diagram in a dispatcher centre and each traffic dispatcher has its own copy. The application supports this convention using client-server architecture when there is one instance of the application running on the server and users (mainly dispatchers) can view current state of the diagram, they can modify it with their permissions etc.

The advantage of this architecture is that any authorized person with access to corporate network can use it. This eliminates the need of installing (or updating) copies on each computer in the company.

The disadvantages of the client-server architecture could be some time lags, relatively difficult implementation, architecture design and used technologies, debugging and deployment. There is also a risk of incompatibility on the server side during the development (e.g. different versions of application on the server).

Data persist in an Oracle database which is connected with business logic using JDBC connector and Hibernate object-relational mapping. Client's requirements are implemented using RPC (remote procedure call, serialized messages). The client part of the application is implemented using JavaScript. JavaScript code was compiled from Java source code using GWT framework. The simplified architecture diagram is on Figure 2. 


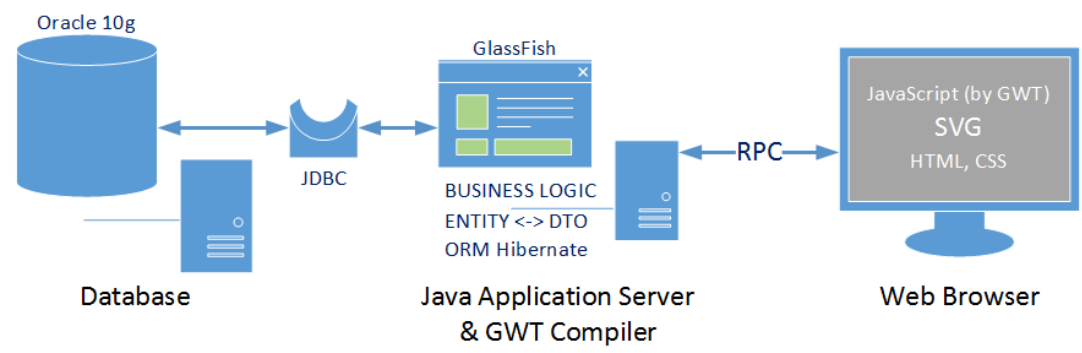

Figure 2: Simplified architecture of application.

\section{Application implementation}

This section presents the key implementation elements that the whole application is made up of - e.g. data import, calendar processing, train's journeys dates and graphical user interface.

\subsection{Data import}

The application uses data that are available in internal systems of SZDC. Data correspond to the railway station Prague main station. Thanks to this the main part of this application was tuned directly for these source data - application supports data import from CSV file to the database. When another station is used then it is necessary to modify this part of application for a specific imported data and edit visual part (such as arrival and departure directions, number and naming of running tracks, etc.).

\subsection{Train calendar detection}

In the data there is also available information about operational dates for all trains in text form (this notation is commonly used in timetables). There is also additional information, such as "train operates as needed", train runs along occupied track in the station, "booster train", etc.

Most of the calendar data consists of information about the train's operational days within a year. It is usually stated in notes for each train individually. If the note contains no such an information then the train operates every day for the whole period of TTD. The structure of a text note to clarify the train calendar is established by SZDC and machine processing is quite difficult. To illustrate the content of the notation, an example of specifying calendar of the train 19051, is given below:

operates in ! 6 and !+ till 17.III. and from 2.XI., from 23.III. till 26.X. operates in !6 a 1., 8.V., 5.VII., doesn't operate 6.VII./operates in ! 6 and !+

A note may include pictograms in text format that is commonly used in timetables. Ahead of this pictogram in text format there is always the exclamation mark followed by wildcard character. 
For recognition of train calendar notes there was implemented heuristic algorithm that makes it possible to detect most of all notes and transfer them to a bit representation. This makes it possible to filter trains for any given dates.

To handle Czech calendar easily there were implemented methods for identification working days, holidays (including Easter floating date) for current year or given interval of days, then convert them to a bit representation and then save them as a calendar for a given train.

Some more complex and very specific cases in calendar representation in source data was not recognized so the calendar was set to default calendar without any additional details. The original calendar (text form notes) can be displayed in train's detail in diagram view as well as in a tabular train summary.

Thanks to recognized calendars it is possible to filter trains by date which is great benefit mainly for clarity of the diagram. At default there are displayed trains for a particular day - actual day (with an option to display any day or show all trains).

\section{User interface}

Design of the user interface (UI) is based on the application requirements. It is quite limited by the fact that the user part of the application is running in a web browser. UI of the application was designed with focus on simplicity, clarity and usability.

There is simple user panel at the top of user interface and list of available tabbed panels for displaying individual components (list of all trains in table - version for read only and editable version, main graphical module with track occupation diagram, excluded trains and running tracks, list of realised changes, user control panel, etc.). User interface is assembled dynamically when a user logs into application, so appearance of application depends on the role of logged in user. Figure 3 depicts part of the main application module - rendered track occupation diagram with vector graphics and a detail of custom menu that allows user to perform editing.

\section{Conclusion}

The aim of this paper is to present the actual development of a supporting tool for dispatchers at passenger railway station which is in this particular case a web application for track occupation diagram management.

The presented application is fully functional in prototype version and contains defined requirements that are listed in section 3.

For deploying this application to a commercial environment it would be needed to review some parts of the application or even add some further requested features (such as export of track occupation diagram for given day in a defined format e.g. PDF). However, these requirements were not defined for the development of prototype version of the application because they do not form the whole core of solved problems and therefore they have not been implemented yet. 


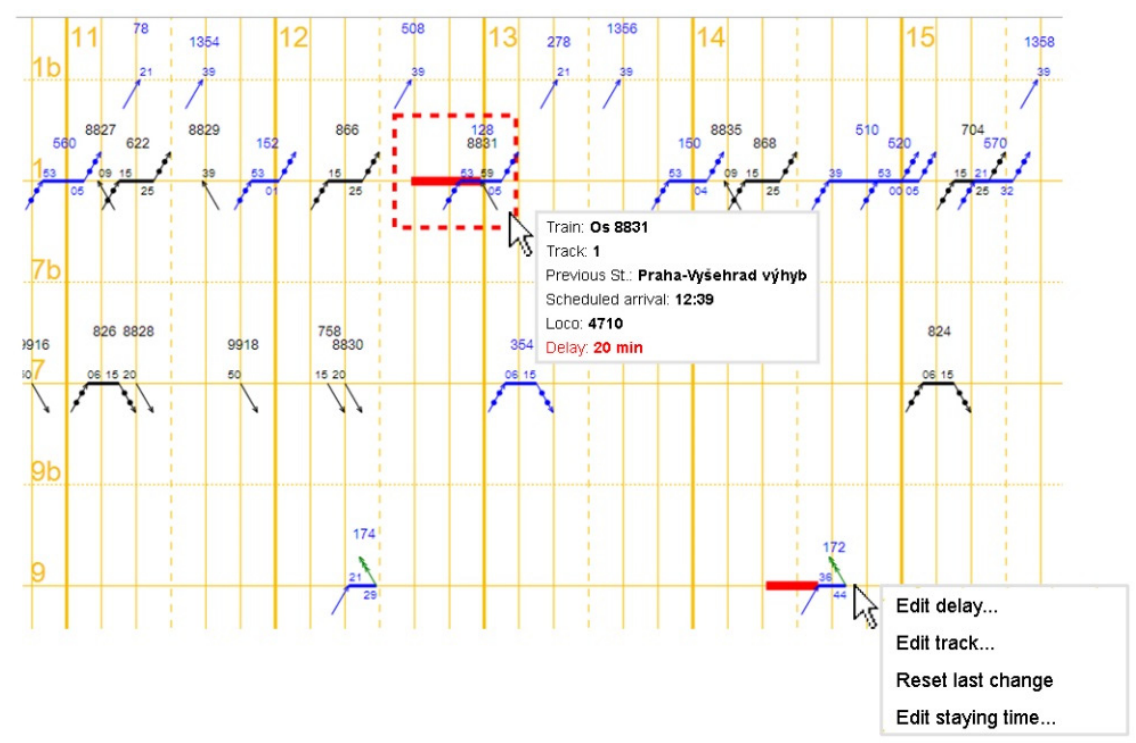

Figure 3: Part of the diagram and detail of user context menu.

\section{References}

[1] Regulation ČD D5: Technological procedures for railway station Prague main station: Czech Railways, 2007.

[2] Hejzlar, J. Track occupancy plans for passenger railway stations. Pardubice: University of Pardubice, Jan Perner Transport Faculty 2007.

[3] Štekr, K., Cibulka, F. Track occupation diagram for railway station Prague main station for TTD 2012/2013. Prague: Czech Railways, 2013. 